\title{
Transcritical Bifurcations and Algebraic Aspects of Quadratic Multiparametric Families
}

\author{
${ }^{\mathrm{a} O R G E}$ RODRÍGUEZ CONTRERAS, ${ }^{\mathrm{b}}$ ALBERTO REYES LINERO, \\ ${ }^{\mathrm{c} B L A D I M I R}$ BLANCO MONTES, ${ }^{\mathrm{d}}$ PRIMITIVO B. ACOSTA HUMÁNEZ \\ ${ }^{a, b, c}$ Facultad de Ciencias Básicas Universidad del Atlántico, Barranquilla- COLOMBIA \\ ${ }^{a}$ Departamento de Matemáticas y Estadística Universidad del Norte; \\ ${ }^{d}$ Facultad de Ciencias Básicas y Biomédicas, Universidad Simón Bolívar, Barranquilla - COLOMBIA \\ ${ }^{d}$ Instituto Superior de Formación Docente Salomé Ureña, Recinto Emilio Prud'Homme, \\ Santiago -DOMINICAN REPUBLIC
}

\begin{abstract}
This article reveals an analysis of the quadratic systems that hold multiparametric families therefore, in the first instance the quadratic systems are identified and classified in order to facilitate their study and then the stability of the critical points in the finite plane, its bifurcations, stable manifold and lastly, the stability of the critical points in the infinite plane, afterwards the phase portraits resulting from the analysis, moreover Algebraic aspects are also included such that hamiltonian cases and Galois differential groupes. It should be noted that these families have associated oscillating type problems given their similarity to the Liénard equations.
\end{abstract}

Key-Words: Quadratic Polynomial Systems, Critical Points, Bifurcations, Stable Manifold, Phase portraits.

Received: January 5, 2021. Revised: April 4, 2021. Accepted: April 17, 2021. Published: May 15, 2021.

\section{Introduction}

Systems of differential equations are known to express a number of mathematical, physical and engineering situations. In particular, this article is based about the study of all quadratic multiparametric subfamilies associated with the next family: Given the family with $a, b, c, m, k \in \mathbb{R}$.

$\left\{\begin{array}{l}\dot{x}=y \\ \dot{y}=\left(\alpha x^{m+k-1}+\beta x^{m-k-1}\right) y-\gamma x^{2 m-2 k-1}\end{array}\right.$

We can find antecedents of the algebraic and qualitative studies of this family in [1, 2, 3]. In general, we can see qualitative studies about planar systems in [4], furthermore antecedents of applied bifurcations study in [5]. In the present work, we take Proposition 4.1, pag 12, in [2, 3] which the goal of analyze each quadratic subfamily equivalently to (1.1). Considering the constants $a, b, c$, and $s, p, r \in \mathbb{Z}^{+}$. Then, we analyze different cases to determine quadratic systems attached to (1.1) taking into account the regions in the space determined by the for the different parameters.

For the study of the quadratic multiparametric families described by (1.1) where we use different topics studied in [6, 12, 13] and [7]. Then, we find the critical points associated with each quadratic family and analyzing their stability in both the finite and infinite planes, also we present a deeper study study determined by regions to see the changes in stability of the critical points and from here analyze bifurcations presented in some families.

Definition 1.1 (Saddle-focus-saddle Bifurcations). We will call a Bifurcations saddle-focus-saddle is when a parameter change it implies that two critical points, one saddle, collapse in a focus and later they recover its original stability.

\section{Conditions For The Problem.}

The following section allows us to identify the quadratic cases associated to (1.1).

\subsection{Reduction to 5 Families}

The next proposition is a particular case of proposition 4.1 in [3], we inly consider the quadratic cases.

Proposition 2.1. Let $a, b, c, m, k \in \mathbb{R} y s, p, r \in \mathbb{Z}^{+}$. Quadratic systems associated with each subfamily of 1.1) are equivalently to the following families:

$$
\begin{aligned}
& \mathbf{I}:\left\{\begin{array}{l}
\dot{x}=y \\
\dot{y}=-c x^{2}
\end{array}\right. \\
& \text { II: }\left\{\begin{array}{l}
\dot{x}=y \\
\dot{y}=2 b y x
\end{array}\right. \\
& \text { III: }\left\{\begin{array}{l}
\dot{x}=y \\
\dot{y}=2 a y x
\end{array}\right. \\
& \boldsymbol{I V}:\left\{\begin{array}{l}
\dot{x}=y \\
\dot{y}=a\left(\frac{p+4}{2}\right) y-\frac{3}{2} a^{2} x-c x^{2}
\end{array}\right.
\end{aligned}
$$




$$
\boldsymbol{V}:\left\{\begin{array}{l}
\dot{x}=y \\
\dot{y}=b\left(\frac{s+4}{2}\right) y-\frac{3}{2} b x-c x^{2}
\end{array}\right.
$$

Proof. We analyze each subfamily of the system (1.1), where We observe the different possibilities for the constants $a, b$ and $c$, are equal to 0 . Some of cases are:

I. For $a=0, b=0$ and $c \neq 0$. If $s=0$, then $p=1$. If $s=1$, then $p=0$.

II. For $a \neq 0, b \neq 0$ and $c \neq 0$. We observed that $\operatorname{deg}(Q)=\max \{2 p+1,2 s+1, s+p+1\}$.

Case 1. If $\operatorname{deg}(Q)=2 p+1$, then $2 p+1=2$ so $p=\frac{1}{2} \notin \mathbb{Z}^{+}$.

Case 2. If $\operatorname{deg}(Q)=2 s+1$, then $2 s+1=2$ so $s=\frac{1}{2} \notin \mathbb{Z}^{+}$.

Case 3. If $\operatorname{deg}(Q)=s+p+1$, then we return to reasoning in the family $\mathbf{I}$, so $s=0$ then $p=1$, but we have that $2 p+1=3$ and this case would be cubic. Same for $p=0$ and $s=1$.Therefore, this family does not have quadratic cases.

\section{Finite Plane}

Proposition 3.1. $(0,0)$ is a cusp of family (2.1) .

Proof. The critical point associated with the system (2.1) is $(0,0)$. The eigenvalues associate to the Jacobian matrix $D F(0,0)$ are $\lambda_{2}=\lambda_{2}=0$. Then, according to the Theorem ([7, pág 116]), where $A(x, y)=0$ and $y=0$, on the other hand We have to $B(x, y)=-c x^{2}$,we get that $F(x)=-c x^{2}$ and $G(x)=0$. Therefore the origin of the system (2.1) is a cusp.

Proposition 3.2. The system (2.2) have infinite critical points.

Proof. $(x, 0)$ which is a line of critical points associated with the system (2.2). We see the solution:

$$
y=b x^{2}+k \text {, where } k \text { is a constant. }
$$

Proposition 3.3. The system (2.3) have infinite critical points.

Proof. $(x, 0)$ which is a line of critical points associated with the system (2.3). We see the solution:

$$
y=a x^{2}+k, \text { where } k \text { is a constant. }
$$

Proposition 3.4. a) The point $(0,0)$ is an stable node if $a<0$ and unstable if $a>0$, and $\left(\frac{-3 a^{2}}{2 c}, 0\right)$ is a saddle. b) If $p=0,(0,0)$ is an stable focus if $a<0$ and unstable if $a>0$, and $\left(\frac{-3 a^{2}}{2 c}, 0\right)$ is a saddle.

Proof. Critical points associated with the system (2.4) are: $(0,0)$ and $\left(\frac{-3 a^{2}}{2 c}, 0\right)$.

Let $d=a(p+4)$

a. For the Jacobian matrix $D F(0,0)$, eigenvalues are:

$\lambda_{1}=\frac{1}{4}\left[d+\sqrt{d^{2}-24 a^{2}}\right]$ and $\lambda_{2}=$ $\frac{1}{4}\left[d-\sqrt{d^{2}-24 a^{2}}\right]$. According to the Theorem ([7, pág 71]) we see that $\lambda_{1} \lambda_{2}>0$ therefore $(0,0)$ is an stable node if $a<0$ and unstable if $a>0$.

Now, for the Jacobian matrix $D F\left(\frac{-3 a^{2}}{2 c}, 0\right)$, eigenvalues are:

$\lambda_{1}=\frac{1}{4}\left[+\sqrt{d^{2}+24 a^{2}}\right]$ y $\quad \lambda_{1}=$ $\frac{1}{4}\left[d-\sqrt{d^{2}+24 a^{2}}\right]$. According to the Theorem ([7, pág 71]) we see that $\lambda_{1} \lambda_{2}<0$, then $\left(\frac{-3 a^{2}}{2 c}, 0\right)$ is a saddle.

b. If $p=0$, for the Jacobian matrix $D F(0,0)$ eigenvalues are:
$\lambda_{1}=\frac{a}{2}(2+i \sqrt{2})$ and $\lambda_{2}=\frac{a}{2}(2-i \sqrt{2})$. According to the Theorem ([7, pág 71]) we see that $(0,0)$ is an stable focus if $a<0$ and unstable if $a>0$.

Now, for the Jacobian matrix $D F\left(\frac{-3 a^{2}}{2 c}, 0\right)$, eigenvalues are:

$\lambda_{1}=\frac{a}{4}[4+2 \sqrt{10}]$ and $\lambda_{1}=\frac{a}{4}[4-2 \sqrt{10}]$. According to the Theorem ([7, pág 71]) we see that $\lambda_{1} \lambda_{2}<0$, then $\left(\frac{-3 a^{2}}{2 c}, 0\right)$ is a saddle.

Before looking at the following proposition, We define the following regions:

$$
\begin{aligned}
& R_{1}=\left\{(b, c, d) \in \mathbb{R}^{3} \mid d^{2}-24 b>0\right\} \\
& R_{2}=\left\{(b, c, d) \in \mathbb{R}^{3} \mid d^{2}-24 b=0\right\} \\
& R_{3}=\left\{(b, c, d) \in \mathbb{R}^{3} \mid d^{2}-24 b<0, c>0\right\} \\
& R_{4}=\left\{(b, 0, d) \in \mathbb{R}^{3} \mid d^{2}-24 b>0\right\} \\
& R_{5}=\left\{(b, 0, d) \in \mathbb{R}^{3} \mid d^{2}-24 b=0\right\} \\
& R_{6}=\left\{(b, 0, d) \in \mathbb{R}^{3} \mid d^{2}-24 b<0\right\} \\
& R_{7}=\left\{(0, c, 0) \in \mathbb{R}^{3} \mid c>0\right\} \\
& R_{8}=\left\{(b, c, d) \in \mathbb{R}^{3} \mid c<0\right\}
\end{aligned}
$$

We note that $\mathbb{R}^{3}=\bigcup_{i=1}^{8} R_{i}$. Now in $R_{3}$ and $R_{4}$ we consider the following subsets:

$$
\begin{aligned}
& E_{1}=\left\{(b, c, d) \in \mathbb{R}^{3} \mid d^{2}-24 b<0, d>0, c>0\right\} \\
& E_{2}=\left\{(b, c, d) \in \mathbb{R}^{3} \mid d^{2}-24 b<0, d<0, c>0\right\} \\
& E_{3}=\left\{(b, c, d) \in \mathbb{R}^{3} \mid d^{2}+24 b<0, d>0, c>0\right\}
\end{aligned}
$$




$$
\begin{aligned}
& E_{4}=\left\{(b, c, d) \in \mathbb{R}^{3} \mid d^{2}-24 b<0, d<0, c>0\right\} \\
& E_{5}=\left\{(b, c, d) \in \mathbb{R}^{3} \mid d^{2}-24 b<0, d>0, c<0\right\} \\
& E_{6}=\left\{(b, c, d) \in \mathbb{R}^{3} \mid d^{2}-24 b<0, d<0, c<0\right\} \\
& E_{7}=\left\{(b, c, d) \in \mathbb{R}^{3} \mid d^{2}+24 b<0, d>0, c<0\right\} \\
& E_{8}=\left\{(b, c, d) \in \mathbb{R}^{3} \mid d^{2}-24 b<0, d<0, c<0\right\} \\
& E_{9}=\left\{(b, c, d) \in \mathbb{R}^{3} \mid d^{2}-24 b>0, d>0, c>0\right\} \\
& E_{10}=\left\{(b, c, d) \in \mathbb{R}^{3} \mid d^{2}-24 b>0, d<0, c>0\right\} \\
& E_{11}=\left\{(b, c, d) \in \mathbb{R}^{3} \mid d^{2}-24 b>0, d<0, c<0\right\} \\
& E_{12}=\left\{(b, c, d) \in \mathbb{R}^{3} \mid d^{2}-24 b>0, d>0 c<0\right\}
\end{aligned}
$$

Proposition 3.5. Let the family (2.5) with $(b, c, d) \in$ $\mathbb{R}^{3}$, then:

a) If $(b, c, d) \in R_{1}$ and $b>0$ then the point $(0,0)$ is unstable node and the point $\left(\frac{-3 b}{2 c}, 0\right)$ is a saddle. if $b<0$ then the point $(0,0)$ is a saddle and the point $\left(\frac{-3 b}{2 c}, 0\right)$ is stable node.

b) If $(b, c, d) \in R_{2}$ and $b>0$, then the critical point $(0,0)$ is a unstable node and the critical point $\left(\frac{-3 b}{2 c}, 0\right)$ is saddle.

c) If $(b, c, d) \in R_{3}$ and $b>0$ then the point $(0,0)$ is stable focus and the point $\left(\frac{-3 b}{2 c}, 0\right)$ is a saddle. If $b<0$ then point $(0,0)$ is a unstable focus and the point $\left(\frac{-3 b}{2 c}, 0\right)$ is a unstable node.

Proof. Let $d=b(s+4)$, so critical points associated with the system (2.5) are: $(0,0)$ and $\left(\frac{-3 b}{2 c}, 0\right)$. For the Jacobian matrix $D F(0,0)$, the eigenvalues are:

$$
\begin{gathered}
\lambda_{1}=\frac{1}{4}\left[d+\sqrt{d^{2}-24 b}\right] \text { and } \\
\lambda_{2}=\frac{1}{4}\left[d-\sqrt{d^{2}-24 b}\right] .
\end{gathered}
$$

Now, for $D F\left(\frac{-3 b}{2 c}, 0\right)$, we have the eigenvalues:

$$
\begin{gathered}
\lambda_{1}=\frac{1}{4}\left[d+\sqrt{d^{2}+24 b}\right] \text { and } \\
\lambda_{2}=\frac{1}{4}\left[d-\sqrt{d^{2}+24 b}\right] .
\end{gathered}
$$

a. If $(b, c, d) \in R_{1}$ that is $d^{2}-24 b>0$, for $(0,0)$ We see that $\lambda_{1} \lambda_{2}=\frac{-3 b}{2}$ and $\lambda_{1}>0$, then according to the Theorem ([7, pág 71]) the critical point $(0,0)$ is unstable node if $b>0$ and a saddle if $b<0$.

Now, for $\left(\frac{-3 b}{2 c}, 0\right)$, we have that $d^{2}+24 b>48 b$, then:

1. If $b>0$, According to the Theorem ([7], pág 71]) We see that $\lambda_{1} \lambda_{2}=\frac{-3 b}{2}$, and $b>0$ then $\left(\frac{-3 b}{2 c}, 0\right)$ is a saddle. If $b<0, \lambda_{2}<0$, then stable node.

2. If $b<0$ and $d^{2}+24 b \in[48 b, 0)$, then the critical point $\left(\frac{-3 b}{2 c}, 0\right)$ is stable focus.
3. If $b<0$ and $d^{2}+24 b \geq 0$, then the critical point $\left(\frac{-3 b}{2 c}, 0\right)$ if stable node.

b. If $(b, c, d) \in R_{2}$ that is $d^{2}-24 b=0$ this leans to $b \geq 0$ for $D F(0,0) \lambda_{1}=\lambda_{2}=\frac{d}{4}$, if $b>0$ then the critical point is unstable node. We note that if $b=0$, then this corresponding to (2.1). Now, if $b>0$ that is $d^{2}-24 b>0$, Furthermore $\lambda_{1} \lambda_{2}=\frac{-3 b}{2}$ according to the Theorem ([7], pág 71]) the critical point $\left(\frac{-3 b}{2 c}, 0\right)$ is saddle.

c. If $(b, c, d) \in R_{3}$, that is $d^{2}-24 b<0$ then $b>$ 0 . For $\operatorname{DF}(0,0)$ eigenvalues are: $\lambda_{1}=\frac{1}{4}(d+$ $\left.i \sqrt{24 b-d^{2}}\right)$ and $\lambda_{2}=\frac{1}{4}\left(d-i \sqrt{24 b-d^{2}}\right)$, then according to the Theorem ([7, pág 71]), $(0,0)$ is a focus unstable. Now, for $b>0$ We see that $d^{2}+$ $24 b>0$, that is for $D F\left(\frac{-3 b}{2 c}, 0\right)$, we have that $\lambda_{1,2} \in \mathbb{R}$. So, $\lambda_{1} \lambda_{2}=\frac{-3 b}{2}$, so According to the Theorem ([7, pág 71]) we have that the critical point $\left(\frac{-3 b}{2 c}, 0\right)$ is a saddle.

Proposition 3.6. Given the family (2.5) with $c=0$, then:

a) If $(b, 0, d) \in R_{4}$ and $b>0$, then the critical point $(0,0)$ is a saddle. If $b<0$, then the critical point $(0,0)$ is a stable node.

b) If $(b, 0, d) \in R_{5}$ and $b>0$, then the critical point $(0,0)$ is a unstable node.

c) If $(b, 0, d) \in R_{6}$ and $b>0$, then the critical point $(0,0)$ is a unstable focus. If $b<0$, then the critical point $(0,0)$ is a stable focus.

Proof. With $c=0$, the family (2.5) have the form:

$$
\left\{\begin{array}{l}
\dot{x}=y \\
\dot{y}=\frac{d}{2} y-\frac{3}{2} b x
\end{array}\right.
$$

Here, we see that the only critical point associated with the family (3.1) is $(0,0)$ then, for the Jacobian matrix $D F(0,0)$ the eigenvalues are:

$\lambda_{1}=\frac{1}{4}\left[d+\sqrt{d^{2}-24 b}\right]$ and $\lambda_{2}=\frac{1}{4}[d-$ $\left.\sqrt{d^{2}-24 b}\right]$.

a. If $(b, 0, d) \in R_{4}$ that is $d^{2}-24 b>0$, we have that $\lambda_{1} \lambda_{2}=\frac{-3 b}{2}$, then if $b>0$ according to the Theorem ([7, pág 71]) the critical point $(0,0)$ is a saddle and if $b<0$ and $\lambda_{1}<0$ then the critical point $(0,0)$ is a stable node.

b. If $(b, 0, d) \in R_{5}$ that is $d^{2}-24 b=0$, we have that $\lambda_{1} \lambda_{2}=\frac{d}{4}$, then according to the Theorem ([7, pág 71]) the critical point $(0,0)$ is a unstable node. 
c. If $(b, 0, d) \in R_{6}$ that is $d^{2}-24 b<0$, we have that $\lambda_{1}=\frac{1}{4}\left(d+i \sqrt{24 b-d^{2}}\right)$ and $\lambda_{2}=$ $\frac{1}{4}\left(d-i \sqrt{24 b-d^{2}}\right)$, then if $b>0$ according to the Theorem ([7, pág 71]) the critical point $(0,0)$ is a unstable focus and if $b<0$ and $\lambda_{1}<0$ then the critical point $(0,0)$ is a stable focus.

Now, We will look the stable manifold for the associated systems.

Proposition 3.7. The stable manifold associated with the system (2.4) at the point $\left(\frac{-3 a^{2}}{2 c}, 0\right)$ is:

$$
S: y=\frac{c\left(x+\frac{3 a^{2}}{2 c}\right)^{2}}{(v-w)(v-2 w)}
$$

Proof. Let is observe the stability of the system (2.4) in the point $\left(\frac{-3 a^{2}}{2 c}, 0\right)$ :

Let is look at the eigenvalues in the Jacobian matrix of the system (2.4) $D F\left(\frac{-3 a^{2}}{2 c, 0}\right)$, are

$w=\lambda_{1}=\frac{1}{4}\left[d+\sqrt{d^{2}+24 a^{2}}\right]$ and $v=\lambda_{2}=$ $\frac{1}{4}\left[d-\sqrt{d^{2}+24 a^{2}}\right]$.

that is, $B(x)=C^{-1} A C=\left[\begin{array}{cc}w & 0 \\ 0 & v\end{array}\right], b=\left[\begin{array}{c}b_{1} \\ 0\end{array}\right]$

$$
\begin{aligned}
& F(x)=\left[\begin{array}{c}
0 \\
-c x^{2}
\end{array}\right], \quad G(x)=\frac{c x^{2}}{v-w}\left[\begin{array}{c}
1 \\
-1
\end{array}\right], \\
& U(t)=\left[\begin{array}{ll}
e^{w t} & 0 \\
0 & 0
\end{array}\right], V(t)=\left[\begin{array}{cc}
0 & 0 \\
0 & e^{v t}
\end{array}\right],
\end{aligned}
$$

Then,

$$
\begin{aligned}
& u^{(0)}(t, b)=0, \quad u^{(1)}(t, b)=\left[\begin{array}{c}
e^{w t} b_{1} \\
0
\end{array}\right] \\
& u^{(2)}(t, b)=\left[\begin{array}{c}
e^{w t} b_{1}+\frac{b_{1}^{2} c e^{2 w t}\left[e^{w t}-1\right]}{w(v-w)} \\
\frac{\left.b_{1}^{2} c e^{2 w t}-w\right)}{(v-w)(v-2 w)}
\end{array}\right]
\end{aligned}
$$

Therefore, We can approximate by $\psi_{2}\left(b_{1}\right)=b_{1}$, therefore the stable and unstable manifold are:

$$
S: y=\frac{c\left(x+\frac{3 a^{2}}{2 c}\right)^{2}}{(v-w)(v-2 w)} \quad U: x+\frac{3 a^{2}}{2 c}=\frac{2 c y^{2}}{(v-w)((v-2 w)}
$$

Proposition 3.8. For the system (2.5) We have that:

a) If $(b, c, d) \in R_{1}$ and $b<0$, stable manifold at the point $(0,0)$ is:

$$
S: y=\frac{c x^{2}}{(v-w)(v-2 w)}
$$

b) If $(b, c, d) \in\{(x, y, z) / x>0, y \neq 0\}$, then stable manifold at the point $\left(\frac{-3 b}{2 c}, 0\right)$ is:

$$
S: y=\frac{c\left(x+\frac{3 b}{2 c}\right)^{2}}{(v-w)(v-2 w)}
$$

Proof. a) Let is observe the stability of the system (2.5) for $b<0$ at the point $(0,0)$ :

Let is observe the stability of the system $(2.5)$ in the point $(0,0)$. Let, $w=\lambda_{1}=\frac{1}{4}\left[d+\sqrt{d^{2}-24 b}\right]$ and $v=\lambda_{2}=\frac{1}{4}\left[d-\sqrt{d^{2}-24 b}\right]$.

That is, $B(x)=C^{-1} A C=\left[\begin{array}{cc}w & 0 \\ 0 & v\end{array}\right], a=\left[\begin{array}{c}a_{1} \\ 0\end{array}\right]$

$$
\begin{aligned}
& F(x)=\left[\begin{array}{c}
0 \\
-c x^{2}
\end{array}\right], G(x)=\frac{c x^{2}}{v-w}\left[\begin{array}{c}
1 \\
-1
\end{array}\right] \\
& U(t)=\left[\begin{array}{ll}
e^{w t} & 0 \\
0 & 0
\end{array}\right], \quad V(t)=\left[\begin{array}{cc}
0 & 0 \\
0 & e^{v t}
\end{array}\right],
\end{aligned}
$$

then,

$$
\begin{aligned}
& u^{(0)}(t, a)=0, \quad u^{(1)}(t, a)=\left[\begin{array}{c}
e^{w t} a_{1} \\
0
\end{array}\right] \\
& u^{(2)}(t, a)=\left[\begin{array}{c}
e^{w t} a_{1}+\frac{a_{1}^{2} c e^{2 w t}\left[e^{w t}-1\right]}{w(v-w)} \\
\frac{a_{1}^{2} c e^{2 w t}}{(v-w)(v-2 w)}
\end{array}\right]
\end{aligned}
$$

Therefore, We can approximate by $\psi_{2}\left(a_{1}\right)=a_{1}$, therefore the stable manifold can be approximated by, respectively like $x \rightarrow 0$ :

$$
S: y=\frac{c x^{2}}{(v-w)(v-2 w)}, U: x=\frac{2 c y^{2}}{(v-w)((v-2 w)} .
$$

b)Now we will observe the stability of the system (2.5) at the point $\left(\frac{-3 b}{2 c}, 0\right)$, when $b>0$ :

The eigenvalues of the jacobian matrix $\operatorname{DF}\left(\frac{-3 b}{2 c}, 0\right)$ are: $w=\lambda_{1}=\frac{1}{4}\left[d+\sqrt{d^{2}+24 b}\right]$ and $v=\lambda_{2}=\frac{1}{4}\left[d-\sqrt{d^{2}+24 b}\right]$.

$$
\text { So, } \begin{aligned}
B(x) & =C^{-1} A C=\left[\begin{array}{ll}
w & 0 \\
0 & v
\end{array}\right], a=\left[\begin{array}{c}
a_{1} \\
0
\end{array}\right] \\
F(x) & =\left[\begin{array}{c}
0 \\
-c x^{2}
\end{array}\right], G(x)=\frac{c x^{2}}{v-w}\left[\begin{array}{c}
1 \\
-1
\end{array}\right] \\
U(t) & =\left[\begin{array}{ll}
e^{w t} & 0 \\
0 & 0
\end{array}\right], V(t)=\left[\begin{array}{cc}
0 & 0 \\
0 & e^{v t}
\end{array}\right],
\end{aligned}
$$

then,

$$
\begin{gathered}
u^{(0)}(t, a)=0, \quad u^{(1)}(t, a)=\left[\begin{array}{c}
e^{w t} a_{1} \\
0
\end{array}\right] \\
u^{(2)}(t, a)=\left[\begin{array}{c}
e^{w t} a_{1}+\frac{a_{1}^{2} c e^{2 w t}\left[e^{w t}-1\right]}{w(v-w)} \\
\frac{a_{1}^{2} c e^{2 w t}}{(v-w)(v-2 w)}
\end{array}\right]
\end{gathered}
$$

So, we can approximate by $\psi_{2}\left(a_{1}\right)=a_{1}$, therefore the stable manifold and unstable manifold can be approximated by

$$
S: y=\frac{c\left(x+\frac{3 b}{2 c}\right)^{2}}{(v-w)(v-2 w)}, U: x+\frac{3 b}{2 c}=\frac{2 c y^{2}}{(v-w)((v-2 w)}
$$


Like $x \rightarrow 0$.

\section{Bifurcations}

In this section we will analyze the study of the bifurcations of family (2.5)

Proposition 4.1. Let sets $R_{7}$ and $R_{8}$ are transcritical bifurcations for the system (2.5)

Proof. Let $P_{1}:(0,0)$ and $P_{2}:\left(\frac{3 b}{2 c}, 0\right)$ of proposition (4.5). If $(b, c, d) \in E_{3}$ then $P_{1}$ a saddle and $P_{2}$ is a unstable focus, when $(b, c, d) \in R_{7}, P_{1}$ and $P_{2}$, they collapse on one critical point which point is a cusp. So, when $(b, c, d) \in E_{2}$ then $P_{1}$ a unstable focus and $P_{2}$ is a saddle. Similarly, the same behavior is observed when $(b, c, d) \in E_{2}$, then $(b, c, d) \in R_{7}$ and finally $(b, c, d) \in E_{4}$.

Now, Let $P_{1}:(0,0)$ and $P_{2}:\left(\frac{3 b}{2 c}, 0\right)$ of proposition (4.5). If $(b, c, d) \in E_{4}$ then $P_{1}$ a saddle and $P_{2}$ is a stable focus, when $(b, c, d) \in R_{8}, P_{1}$ and $P_{2}$, they collapse on one critical point which point is a cusp. So, when $(b, c, d) \in E_{1}$ then $P_{1}$ a stable focus and $P_{2}$ is a saddle. Similarly, the same behavior is observed when $(b, c, d) \in E_{1}$, then $(b, c, d) \in R_{8}$ and finally $(b, c, d) \in E_{3}$.

Therefore, sets $R_{7}$ and $R_{8}$ are transcritical bifurcations for the system (2.5)

Proposition 4.2. A set $\left\{(b, 0, d) \mid d^{2}-24 b<0\right\}$ is a bifurcations saddle-focus-saddle for the system (2.5).

Proof. For proposition (4.5), if $(b, c, d) \in E_{1}$, the point $P_{1}$ is a stable focus and $P_{2}$ is a saddle. Now, when $(b, c, d) \in\left\{(b, 0, d) \mid d^{2}-24 b<0, d>0\right\}$ for proposition (3), $P_{1}$ and $P_{2}$ they collapse in an unstable focus when $(b, c, d)$ goes the set $E_{5}$, appear again $P_{1}$ and $P_{2}$ like stable focus and a saddle respectively.

Proposition 4.3. A set $\left\{(b, 0, d) \mid d^{2}-24 b<0\right\}$ is a bifurcations saddle-focus-saddle for the system (2.5).

Proof. For proposition (4.5), if $(b, c, d) \in E_{2}$, the point $P_{1}$ is a unstable focus and $P_{2}$ is a saddle. Now, when $(b, c, d) \in\left\{(b, 0, d) \mid d^{2}-24 b<0, d<0\right\}$ for proposition (3), $P_{1}$ and $P_{2}$ they collapse in an stable focus when $(b, c, d)$ goes the set $E_{6}$, appear again $P_{1}$ and $P_{2}$ like unstable focus and a saddle respectively.

Proposition 4.4. Let sets $E_{9}$ and $E_{10}$ are local bifurcations for the system (2.5)

Proof. Let $P_{1}:(0,0)$ of proposition (4.5). If $(b, c, d) \in E_{9}$ then $P_{1}$ is a stable node. Now, when $(b, c, d) \in E_{10}$ the point $P_{1}$ is a unstable node. Therefore, regions $E_{9}$ and $E_{10}$ are local bifurcations for the system (2.5)
Proposition 4.5. Let sets $E_{11}$ and $E_{12}$ are local bifurcations for the system (2.5)

Proof. Let $P_{2}:\left(\frac{3 b}{2 c}, 0\right)$ of proposition (4.5). If $(b, c, d) \in E_{12}$ then $P_{2}$ is a unstable node. Now, when $(b, c, d) \in E_{11}$ the point $P_{2}$ is a stable node. Therefore, regions $E_{11}$ and $E_{12}$ are local bifurcations for the system (2.5)

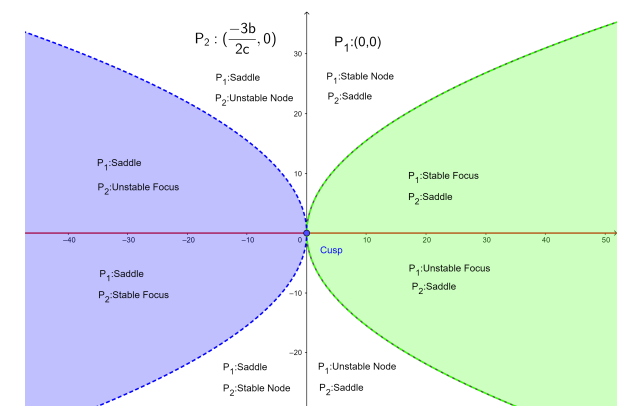

Figure 1: (2.5), $c>0$.

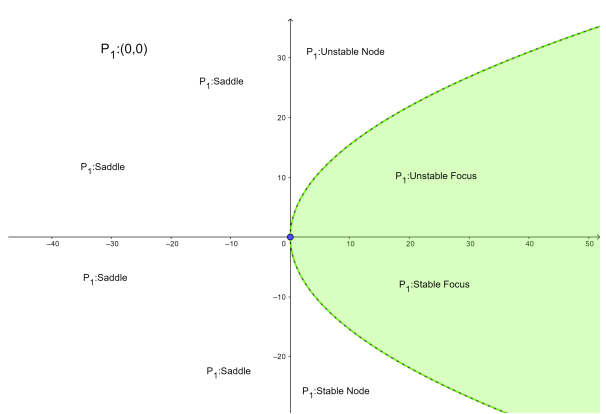

Figure 2: (2.5), $c=0$.

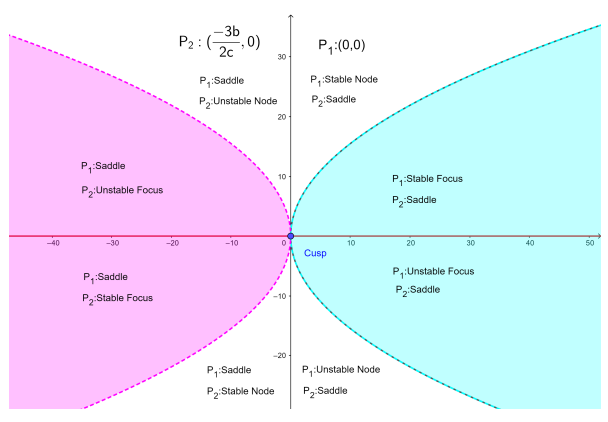

Figure 3: (2.5), $c<0$.

\section{Infinite Plane}

In the Chart $U_{1}$ the associated system for the families $2.1,2.2,2.3,2.4$ and 2.5 respectively are:

$$
\left\{\begin{array}{l}
\dot{u}=-u^{2} v-c \\
\dot{v}=-u v^{2}
\end{array}\right.
$$




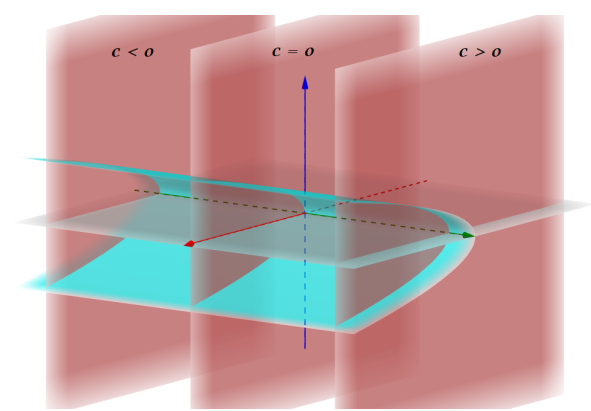

Figure 4: (2.5), Subsets of the space.

$$
\begin{gathered}
\left\{\begin{array}{l}
\dot{u}=-u^{2} v+2 b \\
\dot{v}=-u v^{2}
\end{array}\right. \\
\left\{\begin{array}{l}
\dot{u}=-u^{2} v+2 a \\
\dot{v}=-u v^{2}
\end{array}\right. \\
\left\{\begin{array}{l}
\dot{u}=-u^{2} v+\frac{d u v}{2}-\frac{3 a^{2} v}{2}-c \\
\dot{v}=-u v^{2}
\end{array}\right. \\
\left\{\begin{array}{l}
\dot{u}=-u^{2} v+\frac{d u v}{2}-\frac{3 b v}{2}-c \\
\dot{v}=-u v^{2}
\end{array}\right.
\end{gathered}
$$

The previous systems have not critical points at infinite plane.

In the Chart $U_{2}$ the associated system 2.1:

$$
\left\{\begin{array}{l}
\dot{u}=v+c u^{3} \\
\dot{v}=-c u^{2} v
\end{array}\right.
$$

Proposition 5.1. The point $(0,0)$ is an stable node if $c<0$ and unstable if $c>0$.

Proof. The critical points associated with the system (5.6) is $P:(0,0)$. The eigenvalues associate to Jacobian matrix $D F(0,0)$ are We see that $\lambda_{1}=$ $\lambda_{2}=0$. According to the Theorem ([7, pág 116]), let $v+A(u, v)=0$ a solution of $v+A(u, v)=0$, where $A(u, v)=c u^{3}$ then $v=-c u^{3}$, also we have that $B(u, v)=c u^{2} v$, so $F(u)=-c^{2} u^{5}$ and $G(x)=4 c u^{2}$ then $m=5, n=2, a=-c^{2}, b=4 c$ and $m=2 n+1$, furthermore $b^{2}+4 a(n+1) \geq 0$. Therefore the origin of the system (5.6) in infinite plane is an stable node if $c<0$ and unstable if $c>0$ (5a).

In the Chart $U_{2}$ the associated system (2.2).

$$
\left\{\begin{array}{l}
\dot{u}=v-2 b u^{2} \\
\dot{v}=-2 b u v
\end{array}\right.
$$

Proposition 5.2. The point $(0,0)$ have one hyperbolic and one elliptic sector.
Proof. The critical points associated with the system (5.7) is $P:(0,0)$. The eigenvalues associate to the Jacobian matrix $D F(0,0)$ are, $\lambda_{1}=\lambda_{2}=0$. According to the Theorem ([7], pág 116]), let $v+A(u, v)=0$ a solution of $v+A(u, v)=0$, where $A(u, v)=$ $-2 b u^{2}$ then $v=-2 b u^{2}$, also we have that $B(u, v)=$ $-2 b u v$, so $F(u)=-4 b^{2} u^{3}$ and $G(x)=-6 b u$ then $m=2 n+1$ and $b^{2}+4 a(n+1)$. Therefore the origin of the system (5.7) in infinite plane have one hyperbolic and one elliptic sector (5b).

In the Chart $U_{2}$ the associated system (2.3).

$$
\left\{\begin{array}{l}
\dot{u}=v-2 a u^{2} \\
\dot{v}=-2 a u v
\end{array}\right.
$$

Proposition 5.3. The point $(0,0)$ have one hyperbolic and one elliptic sector.

Proof. The critical points associated with the system (5.8) is $P:(0,0)$. The eigenvalues associate to the Jacobian matrix $D F(0,0)$ are, $\lambda_{1}=\lambda_{2}=0$. According to the Theorem ([7], pág 116]), let $v+A(u, v)=0$ a solution of $v+A(u, v)=0$, where $A(u, v)=$ $-2 a u^{2}$ then $v=-2 a u^{2}$, also we have that $B(u, v)=$ $-2 a u v$, so $F(u)=-4 a^{2} u^{3}$ y $G(x)=-6 a u$ then $m=2 n+1$ and $b^{2}+4 a(n+1)$. Therefore the origin of the system (5.8) in infinite plane have one hyperbolic and one elliptic sector (6a).

In the Chart $U_{2}$ the associated system to (2.4) is:

$$
\left\{\begin{array}{l}
\dot{u}=v-\frac{d u v}{2}+\frac{3}{2} a^{2} u^{2} v+c u^{3} \\
\dot{v}=-\frac{d v^{2}}{2}+\frac{3}{2} a^{2} u v^{2}+c u^{2} v
\end{array}\right.
$$

Proposition 5.4. The point $(0,0)$ is an stable node if $c<0$ and unstable if $c>0$.

Proof. The critical points associated with the system (5.9) is $P:(0,0)$. The eigenvalues associate to the Jacobian matrix $D F(0,0)$ are, $\lambda_{1}=\lambda_{2}=0$. According to the Theorem (([7, pág 116])), Let $v=f(u)$ a solution of $v+A(u, v)=0$ where $v=f(u)=$ $-c u^{3}+\ldots$ an approximation of the Taylor series solution, furthermore $B(u, v)=-\frac{d v^{2}}{2}+\frac{3}{2} a^{2} u v^{2}+c u^{2} v$, then $F(u)=-c^{2} u^{5}+\ldots$ and $G(u)=c u^{2}+\ldots$, so $m=5, n=2, b=4 c$ and $a=-c^{2}$. Therefore the origin in the infinite plane is an stable node if $c<0$ and unstable if $c>0$ (6b).

In the Chart $U_{2}$ the associated system to (2.5) is:

$$
\left\{\begin{array}{l}
\dot{u}=v-\frac{d u v}{2}+\frac{3}{2} b u^{2} v+c u^{3} \\
\dot{v}=-\frac{d v^{2}}{2}+\frac{3}{2} b u v^{2}+c u^{2} v
\end{array}\right.
$$

Proposition 5.5. The point $(0,0)$ is stable node if $c<0$ and unstable if $c>0$. 
Proof. The critical points associated with the system (5.10) is $P:(0,0)$. The eigenvalues associate to the Jacobian matrix $D F(0,0)$ are, $\lambda_{1}=\lambda_{2}=0$. According to the Theorem ([7, pág 116]), let $v=f(u)$ a solution of $v+A(u, v)=0$, where $v=f(u)=$ $-c u^{3}+\ldots$ approximation of the Taylor series solution, furthermore $B(u, v)=-\frac{d v^{2}}{2}+\frac{3}{2} b u v^{2}+c u^{2} v$, then $F(u)=-c^{2} u^{5}+\ldots$ y $G(u)=c u^{2}+\ldots$, so $m=5, n=2, b=4 c$ y $a=-c^{2}$. Therefore the origin in the infinite plane is an stable node if $c<0$ and is a unstable node if $c>0$.

\section{Global Phase Portrait}

In this section We show the global nophase portrait associate to each family:

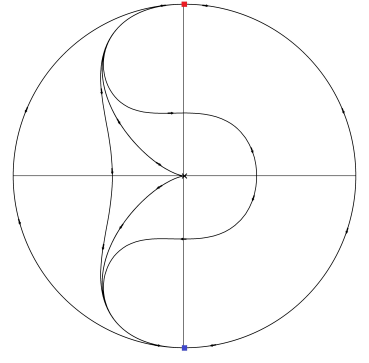

(a) Family I.

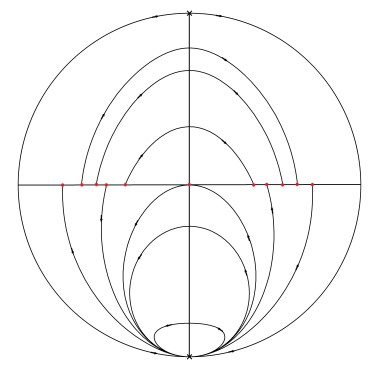

(a) Family III.

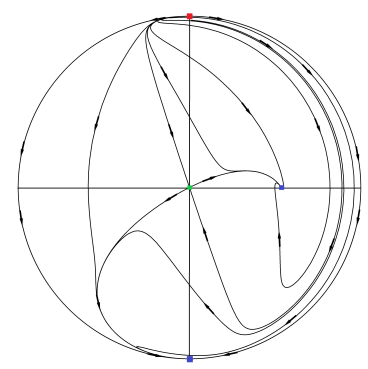

(a) Family V, $b<0$.

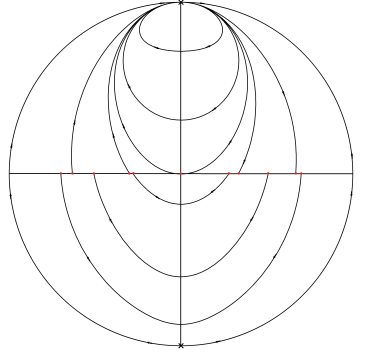

(b) Family II.

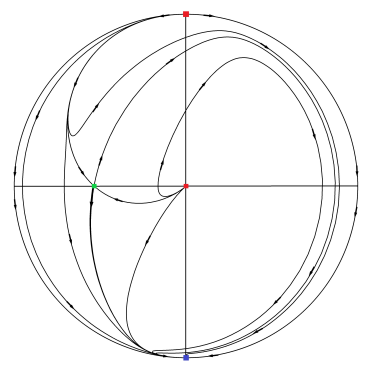

(b) Family IV.

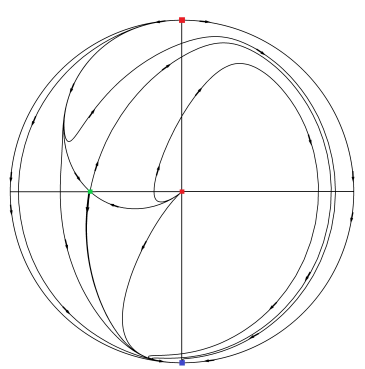

(b) Family V, $b>0$.

\section{Algebraic Aspects}

In this section we follow the references [1, 9, 10, 11]. According to [10, pag. 46], explicit solutions for dif- ferential equation

$$
\frac{d^{2} x}{d t^{2}}=f(x)
$$

are related with the integral curve $(x, \dot{x})$ of the one degree of freedom Hamiltonian system

$$
\dot{x}=y, \dot{y}=f(x), \quad H=\frac{y^{2}}{2}-\int_{x_{0}}^{x} f(\tau) d \tau
$$

Theorem 7.1. Consider the family I, The following statements hold.

1. The dynamical system is hamiltonian with one degree of freedom and with polynomial first integral $H=H(x, y)=\frac{y^{2}}{2}+\frac{c}{3} x^{3}$

2. The integral curve of the Hamiltomian vector field is

$$
\left(-\frac{6}{c} \wp\left(t+k_{0} ; 0,-2 H\right),-\frac{6}{c} \dot{\wp}\left(t+k_{0} ; 0,-2 H\right)\right) .
$$

3. The Differential Galois Group associated to the foliation is isomorphic to $\mathbb{Z}_{2}$.

4. The connected identity component of the Differential Galois Group of the first variational equation along any particular solution is an abelian group.

Proof. We proceed according to each item,

1. The polynomial vector field related with family I is equivalent to Equation (7.1) being $f(x)=$ $-c x^{2}$. In virtue of Equation (7.2) we have the Hamiltonian $H=\frac{y^{2}}{2}+\frac{c}{3} x^{3}$.

2. Due to $y=\dot{x}$, we obtain $y^{2}=-\frac{2 c^{2}}{3}+$ $H$. Through the change of variable $(x, y) \mapsto$ $\left(\sqrt[3]{\frac{-6}{c}} x, \sqrt[3]{\frac{-6}{c}} y\right)$, we arrive to the elliptic curve related with Wierstrass P-function with invariants $g_{2}=0$ and $g_{3}=-2 H$. Thus, the integral curve of the Hamiltonian system is $(x, \dot{x})$, being $x$ given by $-\frac{6}{c} \wp\left(t+k_{0} ; 0,-2 H\right)$.

3. The foliation associated to the vector field of Family I is

$$
y^{\prime}=-\frac{c x^{2}}{y}, \quad,:=\frac{d}{d x} .
$$

Setting $z=\frac{y^{2}}{2}$, we obtain $z^{\prime}=-c x^{2}$ and therefore $z=-\frac{c}{3} x^{3}$. Due to the differential field $K$ is the field of rational functions $\mathbb{C}(x), \sigma(z)=z$ and $\sigma(y)=\lambda \sqrt{z}$, where $\lambda^{2}=1$. Thus, the PicardVessiot extension $L$ is a quadratic extension of $K$ and we can conclude that $\operatorname{DGal}(L / K)$ has two elements. 
4. Let $\left(x_{0}(t), \dot{x}_{0}(t)\right)$ be a particular solution of the polynomial vector field related with Family I. Thus, the first variational equation is

$$
\frac{d}{d t}\left(\begin{array}{l}
\xi_{1} \\
\xi_{2}
\end{array}\right)=\left(\begin{array}{cc}
0 & 1 \\
-2 c x_{0}(t) & 0
\end{array}\right)\left(\begin{array}{l}
\xi_{1} \\
\xi_{2}
\end{array}\right),
$$

which is equivalent to $\ddot{\xi}=-2 c x_{0}(t) \xi$, being $\xi=\xi_{1}$. By Morales-Ramis theory, due to the dynamical system is polynomially integrable, the differential Galois group of this first variational equation is abelian.

Theorem 7.2. Consider the family II, The following statements hold.

1. The first integral of the polynomial vector field is

$$
I=I(x, y)=y-b x^{2}
$$

2. The integral curve of the polynomial vector field is $(x(t), \dot{x}(t))$, where

$$
x(t)=\sqrt{\frac{k_{1}}{b}} \tan \left(\sqrt{k_{1} b\left(k_{2}+t\right)}\right) .
$$

3. The Differential Galois Group associated to the foliation is isomorphic to the identity group.

4. The connected identity component of the Differential Galois Group of the first variational equation around any particular solution is an abelian group.

Proof. We proceed according to each item,

1. The total derivative of $I(x, y)$ vanishes, i.e., $\dot{I}=$ 0 , therefore $I$ is a first integral of the vector field related to family II.

2. Due to $y=\dot{x}$, we obtain $\ddot{x}=b \dot{z}$, where $z=x^{2}$. Thus, $\dot{x}=b x^{2}+k_{1}$, which implies that

$$
\int \frac{d x}{b x^{2}+k_{1}}=t+k_{2}
$$

and then $x(t)=\sqrt{\frac{k_{1}}{b}} \tan \left(\sqrt{k_{1} b\left(k_{2}+t\right)}\right)$.

3. The foliation associated to the vector field of Family II is

$$
y^{\prime}=2 b x, \quad{ }^{\prime}:=\frac{d}{d x} .
$$

Then the solution of this foliation is

$$
y(x)=b x^{2}+k_{1}
$$

- Then we can conclude that $D G a l(L / K)$ has one element, i.e., $\operatorname{DGal}(L / K)=I_{2}$.
4. Let $\left(x_{0}(t), \dot{x}_{0}(t)\right)$ be a particular solution of the polynomial vector field related with Family II. Thus, the first variational equation is

$$
\frac{d}{d t}\left(\begin{array}{l}
\xi_{1} \\
\xi_{2}
\end{array}\right)=\left(\begin{array}{cc}
0 & 1 \\
-2 b y_{0}(t) & 2 b x_{0}(t)
\end{array}\right)\left(\begin{array}{l}
\xi_{1} \\
\xi_{2}
\end{array}\right),
$$

which is equivalent to

$$
\ddot{\xi}-2 b x_{0}(t) \dot{\xi}-2 b y_{0}(t) \xi=0, \xi=\xi_{1} .
$$

Due to the first integral is of polynomial type, by Morales-Ramis theory we can conclude that the connected identity component of the differential Galois group of the first variational equation along any particular solution is an abelian group.

Theorem 7.3. Consider the family III, The following statements hold.

1. The first integral of the polynomial vector field is

$$
I=I(x, y)=y-a x^{2}
$$

2. The integral curve of the polynomial vector field is $(x(t), \dot{x}(t))$, where

$$
x(t)=\sqrt{\frac{k_{1}}{a}} \tan \left(\sqrt{k_{1} a\left(k_{2}+t\right)}\right) .
$$

3. The Differential Galois Group associated to the foliation is isomorphic to the identity group.

4. The connected identity component of the Differential Galois Group of the first variational equation around any particular solution is an abelian group.

Proof. We proceed according to each item,

1. The total derivative of $I(x, y)$ vanishes, i.e., $\dot{I}=$ 0 , therefore $I$ is a first integral of the vector field related to family III.

2. Due to $y=\dot{x}$, we obtain $\ddot{x}=a \dot{z}$, where $z=x^{2}$. Thus, $\dot{x}=a x^{2}+k_{1}$, which implies that

$$
\int \frac{d x}{a x^{2}+k_{1}}=t+k_{2}
$$

and then $x(t)=\sqrt{\frac{k_{1}}{a}} \tan \left(\sqrt{a k_{1}\left(k_{2}+t\right)}\right) .$.

3. The foliation associated to the vector field of Family II is

$$
y^{\prime}=2 a x, \quad ':=\frac{d}{d x} .
$$


Then the solution of this foliation is

$$
y(x)=a x^{2}+k_{1}
$$

- Then we can conclude that $\operatorname{DGal}(L / K)$ has one element, i.e., $\operatorname{DGal}(L / K)=I_{2}$.

4. Let $\left(x_{0}(t), \dot{x}_{0}(t)\right)$ be a particular solution of the polynomial vector field related with Family III. Thus, the first variational equation is

$$
\frac{d}{d t}\left(\begin{array}{l}
\xi_{1} \\
\xi_{2}
\end{array}\right)=\left(\begin{array}{cc}
0 & 1 \\
-2 a y_{0}(t) & 2 a x_{0}(t)
\end{array}\right)\left(\begin{array}{l}
\xi_{1} \\
\xi_{2}
\end{array}\right),
$$

which is equivalent to

$$
\ddot{\xi}-2 a x_{0}(t) \dot{\xi}-2 a y_{0}(t) \xi=0, \xi=\xi_{1} .
$$

Due to the first integral is of polynomial type, by Morales-Ramis theory we can conclude that the connected identity component of the differential Galois group of the first variational equation along any particular solution is an abelian group.

Theorem 7.4. Consider the family $I V$, being $p=-4$. The following statements hold.

1. The dynamical system is hamiltonian with one degree of freedom and with polynomial first integral $H=H(x, y)=\frac{y^{2}}{2}+\frac{c}{3} x^{3}+\frac{3}{4} a^{2} x^{2}$

2. The integral curve of the Hamiltomian vector field is given in terms of P-function.

3. The Differential Galois Group associated to the foliation is isomorphic to $\mathbb{Z}_{2}$.

4. The connected identity component of the Differential Galois Group of the first variational equation along any particular solution is an abelian group.

Proof. We proceed according to each item,

1. The polynomial vector field related with family IV is equivalent to Equation (7.1) being $f(x)=$ $-c x^{2}-\frac{3}{2} a^{2} x$. In virtue of Equation (7.2) we have the Hamiltonian $H=\frac{y^{2}}{2}+\frac{c}{3} x^{3}+\frac{3}{4} a^{2} x^{2}$.

2. Due to $y=\dot{x}$, we obtain $y^{2}=-\frac{2 c^{2}}{3}-\frac{3}{2} a^{2} x+$ $2 H$. Because previous expression is a cubic polynomial in $x$, we can do a suitable change of variable to arrive to the elliptic curve related to Weirstrass P-function with invariants $g_{2}$ and $g_{3}$. Thus, the integral curve of the Hamiltonian system is written in terms of P-function.
3. The foliation associated to the vector field of Family IV is

$$
y^{\prime}=-\frac{c x^{2}-\frac{3}{2} a^{2} x}{y}, \quad,:=\frac{d}{d x} .
$$

Setting $z=\frac{y^{2}}{2}$, we obtain $z^{\prime}=-c x^{2}-\frac{3}{2} a^{2} x$ and therefore $z=-\frac{c}{3} x^{3}-\frac{3}{4} a^{2} x^{2}$. Due to the differential field $K$ is the field of rational functions $\mathbb{C}(x), \sigma(z)=z$ and $\sigma(y)=\lambda \sqrt{z}$, where $\lambda^{2}=1$. Thus, the Picard-Vessiot extension $L$ is a quadratic extension of $K$ and we can conclude that $\operatorname{DGal}(L / K)$ has two elements.

4. Let $\left(x_{0}(t), \dot{x}_{0}(t)\right)$ be a particular solution of the polynomial vector field related with Family IV. Thus, the first variational equation is

$$
\frac{d}{d t}\left(\begin{array}{l}
\xi_{1} \\
\xi_{2}
\end{array}\right)=\left(\begin{array}{cc}
0 & 1 \\
-\frac{3}{2} a^{2}-2 c x_{0}(t) & 0
\end{array}\right)\left(\begin{array}{l}
\xi_{1} \\
\xi_{2}
\end{array}\right),
$$

which is equivalent to $\ddot{\xi}=\left(-\frac{3}{2} a^{2}-2 c x_{0}(t)\right) \xi$, being $\xi=\xi_{1}$. By Morales-Ramis theory, due to the dynamical system is polynomially integrable, the differential Galois group of this first variational equation is abelian.

Theorem 7.5. Consider the family $V$, being $s=-4$. The following statements hold.

1. The dynamical system is hamiltonian with one degree of freedom and with polynomial first integral $H=H(x, y)=\frac{y^{2}}{2}+\frac{c}{3} x^{3}+\frac{3}{4} b x^{2}$

2. The integral curve of the Hamiltomian vector field is given in terms of $P$-function.

3. The Differential Galois Group associated to the foliation is isomorphic to $\mathbb{Z}_{2}$.

4. The connected identity component of the Differential Galois Group of the first variational equation along any particular solution is an abelian group.

Proof. We proceed according to each item,

1. The polynomial vector field related with family $\mathrm{V}$ is equivalent to Equation (7.1) being $f(x)=$ $-c x^{2}-\frac{3}{2} b x$. In virtue of Equation (7.2) we have the Hamiltonian $H=\frac{y^{2}}{2}+\frac{c}{3} x^{3}+\frac{3}{4} b x^{2}$.

2. Due to $y=\dot{x}$, we obtain $y^{2}=-\frac{2 c^{2}}{3}-\frac{3}{2} b x+2 H$. Because previous expression is a cubic polynomial in $x$, we can do a suitable change of variable to arrive to the elliptic curve related to Veirstrass P-function with invariants $g_{2}$ and $g_{3}$. Thus, the integral curve of the Hamiltonian system is written in terms of P-function. 
3. The foliation associated to the vector field of Family V is $y^{\prime}=-\frac{c x^{2}-\frac{3}{2} b x}{y}, \quad,:=\frac{d}{d x}$. Setting $z=\frac{y^{2}}{2}$, we obtain $z^{\prime}=-c x^{2}-\frac{3}{2} b x$ and therefore $z=-\frac{c}{3} x^{3}-\frac{3}{4} b x^{2}$. Due to the differential field $K$ is the field of rational functions $\mathbb{C}(x), \sigma(z)=z$ and $\sigma(y)=\lambda \sqrt{z}$, where $\lambda^{2}=1$. Thus, the Picard-Vessiot extension $L$ is a quadratic extension of $K$ and we can conclude that $\operatorname{DGal}(L / K)$ has two elements.

4. Let $\left(x_{0}(t), \dot{x}_{0}(t)\right)$ be a particular solution of the polynomial vector field related with Family V. Thus, the first variational equation is

$$
\frac{d}{d t}\left(\begin{array}{l}
\xi_{1} \\
\xi_{2}
\end{array}\right)=\left(\begin{array}{cc}
0 & 1 \\
-\frac{3}{2} b-2 c x_{0}(t) & 0
\end{array}\right)\left(\begin{array}{l}
\xi_{1} \\
\xi_{2}
\end{array}\right),
$$

which is equivalent to $\ddot{\xi}=\left(-\frac{3}{2} b-2 c x_{0}(t)\right) \xi$, being $\xi=\xi_{1}$. By Morales-Ramis theory, due to the dynamical system is polynomially integrable, the differential Galois group of this first variational equation is abelian.

\section{Conclusion}

A study was carried out on all possible quadratic cases associated with a family of polynomial systems, such study included the analysis of critical points, their stability, the existence of bifurcations, the existence of Hamiltonian cases and the construction of differential Galois groups.

\section{References:}

[1] P.B. Acosta-Humánez, J.T Lazaro, J.J. MoralesRuiz, Ch. Pantazi, On the integrability of polynomial fields in the plane by means of PicardVessiot theory, Discrete \& Continuous Dynamical Systems-A 35 (2015): 1767-1800.

[2] P. B. Acosta-Humánez, A. Reyes-Linero and J. Rodríguez-Contreras, Algebraic and qualitative remarks about the family $y y^{\prime}=\left(\alpha x^{m+k-1}+\right.$ $\left.\beta x^{m-k-1}\right) y+\gamma x^{2 m-2 k-1}$, arXiv:1807.03551 (2014).

[3] J. Rodríguez-Contreras, P. B. Acosta-Humánez and A. Reyes-Linero, Algebraic and qualitative remarks about the family $y y^{\prime}=\left(\alpha x^{m+k-1}+\right.$ $\left.\beta x^{m-k-1}\right) y+\gamma x^{2 m-2 k-1}$, Open Mathematics 17 (2019), 1220-1238.

[4] M. L. Vílchez Lobato, F. Velasco Morente, J. J. García del Hoyo ,Bifurcaciones transcriticas y ciclos límites en un modelo dinámico de competición entre dos especies. Una aplicación a la pescadera de engraulis encrasicholus de la Región Suratlántica espanñola, (2002).

[5] Valery A. Gaiko, Multiple limit cycle bifurcations of the FitzHugh-Nagumo neuronal model, (2011)

[6] Perko,L., Differential Equations and Dynamical Systems (New York: Springer Verlag), 2001.

[7] Dumortier F., Llibre J., Artes J.,Qualitative Theory Of Planar Differential Systems, 2006.

[8] Acosta-Humánez, P., and G. Jiménez. "Some tastings in Morales-Ramis theory." Journal of Physics: Conference Series. Vol. 1414. No. 1. IOP Publishing, 2019.

[9] Acosta-Humánez, M. F., Acosta-Humánez, P. B., \& Tuirán, E. (2018). Generalized LennardJones Potentials, SUSYQM and Differential Galois Theory. SIGMA. Symmetry, Integrability and Geometry: Methods and Applications, 14, 099.

[10] Acosta-Humánez, P. B. (2006). La teoría de Morales-Ramis y el algoritmo de Kovacic. Lecturas matemáticas, 27(3), 21-56.

[11] Acosta-Humánez, P. B. (2009). Nonautonomous Hamiltonian Systems and Morales-Ramis Theory I. The case $\ddot{x}=f(x, t)$. SIAM Journal on Applied Dynamical Systems, 8(1), 279-297.

[12] I. G. Burova, I. I. Narbutovskikh, E. F. Muzafarova, Image Processing and the Spline Approximation of the Third and Fifth Order, International Journal of Circuits, Systems and Signal Processing, pp. 550-557, Volume 13, 2019.

[13] I. G. Burova, E. F. Muzafarova, Approximations with Polynomial, Trigonometric, Exponential Splines of the Third Order and Boundary Value Problem, International Journal of Circuits, Systems and Signal Processing, pp. 460-473, Volume 14, 2020.

\section{Creative Commons Attribution License 4.0 (Attribution 4.0 International, CC BY 4.0)}

This article is published under the terms of the Creative Commons Attribution License 4.0

https://creativecommons.org/licenses/by/4.0/deed.en US 